\title{
Very Early Onset Steroid-Induced Avascular Necrosis of the Hip and Knee in a Patient with Idiopathic Thrombocytopenic Purpura
}

\author{
Necmettin Yildiz, Fusun Ardic and Saadet Deniz
}

\begin{abstract}
Avascular necrosis (AVN) is a devastating adverse effect of steroid therapy rarely reported in idiopathic thrombocytopenic purpura (ITP). We describe a 64-year-old woman with progressive left knee and hip pain for 30 days resulting with the inability to ambulate. After she had been diagnosed ITP pulse steroid treatment was started in the department of Hematology. Two weeks later she described left knee and left hip pain. On physical examination, motion in her left knee and hip was severely painful without inflammation but range of motion was not limited. Magnetic resonance imaging showed multiple bony infarcts in the proximal femur, distal femur and proximal tibia, consistent with AVN. This case report is the first to define concurrent hip and knee AVN at a very early stage due to steroid use in a patient with ITP.
\end{abstract}

Key words: avascular necrosis, idiopathic thrombocytopenic purpura, steroid

(Inter Med 47: 1989-1992, 2008)

(DOI: 10.2169/internalmedicine.47.1038)

\section{Introduction}

Avascular necrosis (AVN) is in situ death of a segment of bone resulting from the interruption of blood supply. The pathogenesis of this process is not well understood, but several theories have been proposed $(1,2)$.

In the literature, there have been only two cases of AVN due to high-dose steroid use for the treatment of idiopathic thrombocytopenic purpura (ITP) $(3,4)$. Steroid-induced AVN of the hip resulting in femur neck fracture in a patient with ITP was defined by Usui et al (3). Havel et al determined AVN of bilateral lateral femoral condyles due to high dose steroid use in a patient with mixed connective-tissue disease and thrombocytopenic purpura (4). Though it is frequent in different diseases due to steroid use, hip-and-knee concomitant and very early diagnosed AVN in these groups are rare (3-9). The present case report is the first to define concurrent hip and knee AVN at a very early stage due to steroid use in a patient with ITP.

\section{Case Report}

The patient was a 64-year-old woman with a history of 30 days of severe, left knee and hip pain without history of arthritis. She was unable to tolerate weight-bearing on the left lower limb. She denied recent fever or chills. However, 45 days earlier, petechiaes at the upper and lower extremities and oral mucosa had appeared and her platelet count was $4,000 / \mu \mathrm{L}$ therefore she had been diagnosed as ITP in the department of hematology. Though the patient was treated with three units of platelet suspension an increased number of megacaryocytes were observed in bone marrow aspiration material. In this case, pulse steroid therapy was performed. Steroid was given as $1,000 \mathrm{mg}$ /day for the initial 3 days, followed with $500 \mathrm{mg} /$ day for 3 days, and $250 \mathrm{mg} /$ day for 3 days. In the following 3 weeks the steroid dose was gradually decreased. After this treatment petechiae disappeared, but as the platelet count increased up to $12,000 /$ $\mu \mathrm{L}, 60$ gr intravenous immunglobulin was administered and the platelet count increased to $39,000 / \mu \mathrm{L}$. When the patient attended our outpatient clinic, she had been using $8 \mathrm{mg} /$ day 


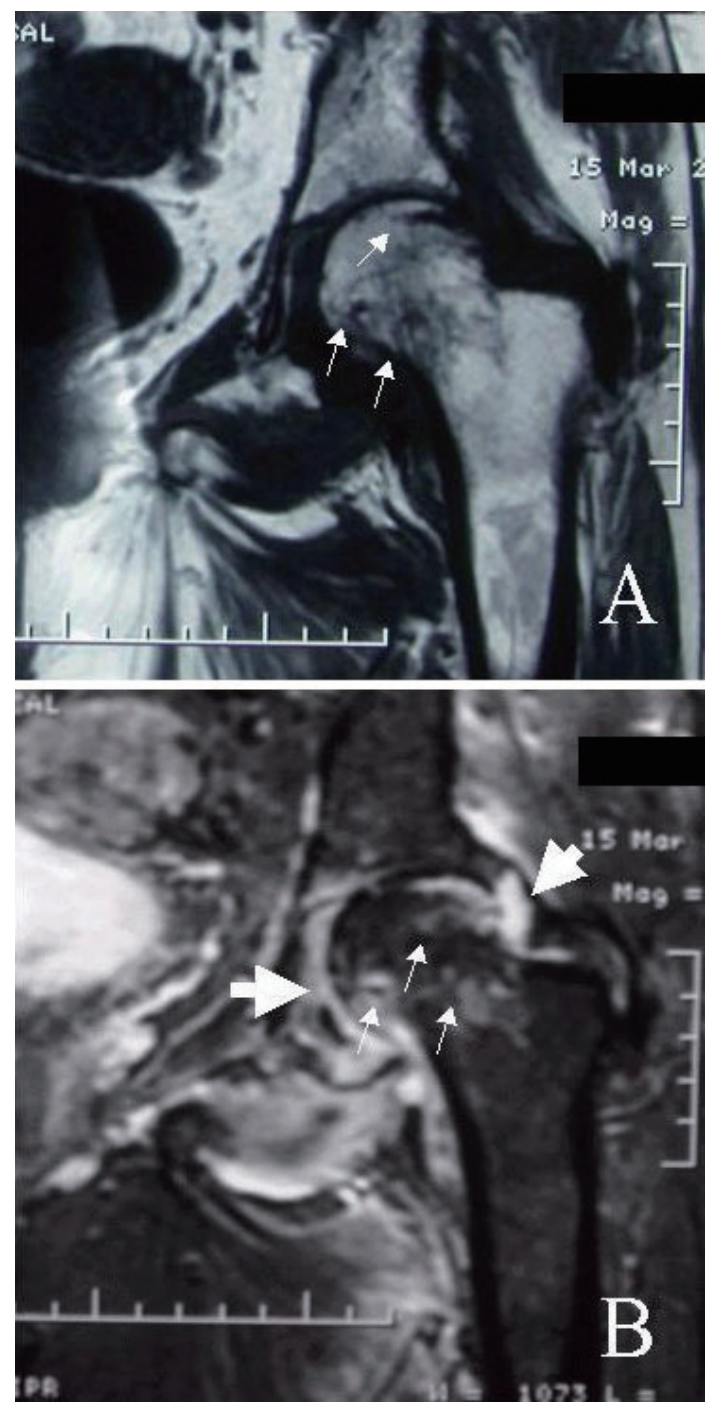

Figure 1. Coronal T1-weighted (A) and T2-weighted (B) MR images of left hip show discrete intramedullary bone marrow abnormalities (small arrows) in left femoral head and neck. T2-weighted (B) MR image showing joint fluid (large arrows) adjacent to the femoral neck.

of steroid.

Two weeks after starting steroid therapy, the patient suddenly suffered from left knee and left hip pain. The patient was not able to walk without support for the last 15 days. She mentioned that her knee and hip pain increased with mobility and decreased during rest. On physical examination, her vital signs were within normal limits. Range of motion was not limited, but her left knee and hip were severely painful. There were no symptoms of inflammation in her painful joints. Tendon reflexes, sensation and strength in both upper and lower extremities were normal. The distal pulses were intact in both lower limbs.

The erythrocyte sedimentation rate was $51 \mathrm{~mm} / \mathrm{h}$, white blood cell count was $8,600 \mathrm{~K} / \mu \mathrm{L}$. Radiographs of both knees and hips were normal. Both of her hips and her left knee joint were checked with magnetic resonance (MR) imaging. MR images of both hips showed osteonecrosis and intramedullary bone marrow abnormalities in the left hip
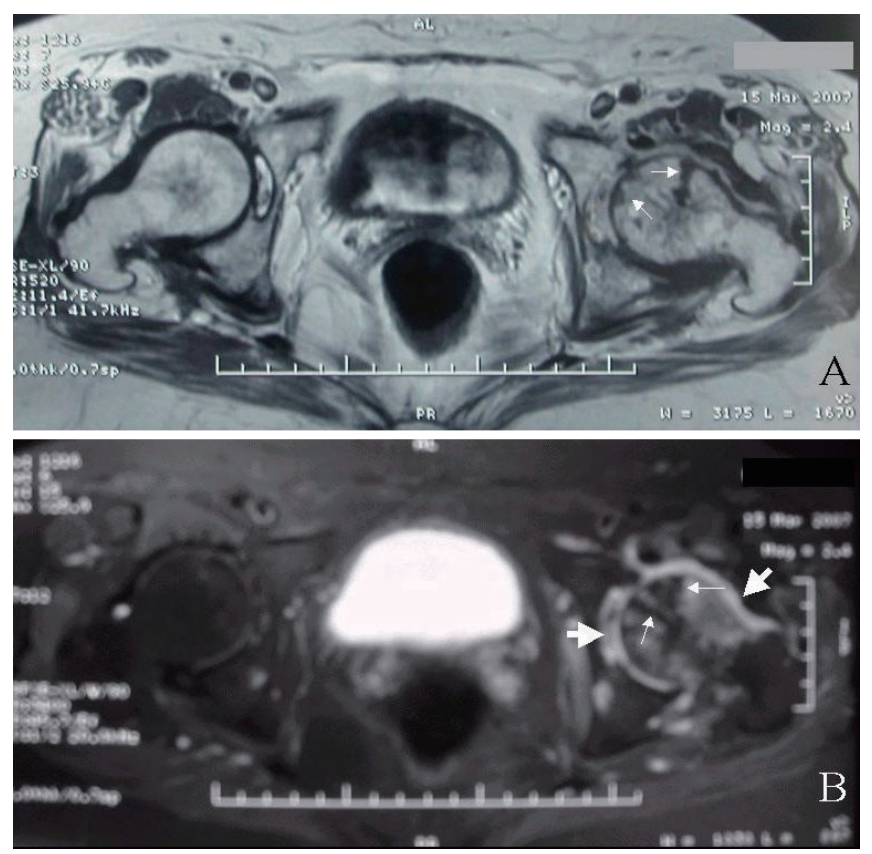

Figure 2. Axial T1-weighted (A) and T2-weighted (B) MR images of both hips show demarcated areas of osteonecrosis and intramedullary bone marrow abnormalities in left femoral head (small arrows). Axial T2-weighted (B) MR image shows joint fluid (large arrows).

(Fig. 1 and Fig. 2). MR images of the left knee showed osteonecrosis in the lateral femoral condyle, medial femoral condyle and tibial plateau (Fig. 3). Depending on the clinical and radiological findings AVN was diagnosed in left hip and left knee.

At the onset of AVN; prothrombin time (PT) was $114.4 \%$ (11.3 seconds), international normalized ratio (INR) was 0.9 and activated partial thromboplastin time (APTT) was 21.6 seconds representing normal values. Initially, the current steroid therapy was terminated. Afterwards pain management, range of motion exercises, strengthening and limited mobility training were applied. Though rehabilitation efforts led to some functional improvement, ultimately ipsilateral total knee and hip arthroplasties were recommended for the treatment of her AVN.

\section{Discussion}

AVN (osteonecrosis) represents bone tissue death because of vascularization failure, and it is a serious side effect of steroid therapy. Unlike many other side effects, osteonecrosis is irreversible and may be extensive $(1,8)$.

Alterations in fat metabolism due to the development of fat emboli in the microcirculature or by fatty infiltration of the marrow is the mostly accepted explanation for the mechanism of steroid-induced AVN. An increase in bone marrow pressure due to intramedullary lipocyte hypertrophy causing decreased bone blood flow and ischemia results with microinfarcts and osteonecrosis $(1,2)$.

Steroid-induced AVN, may develop in patients receiving 

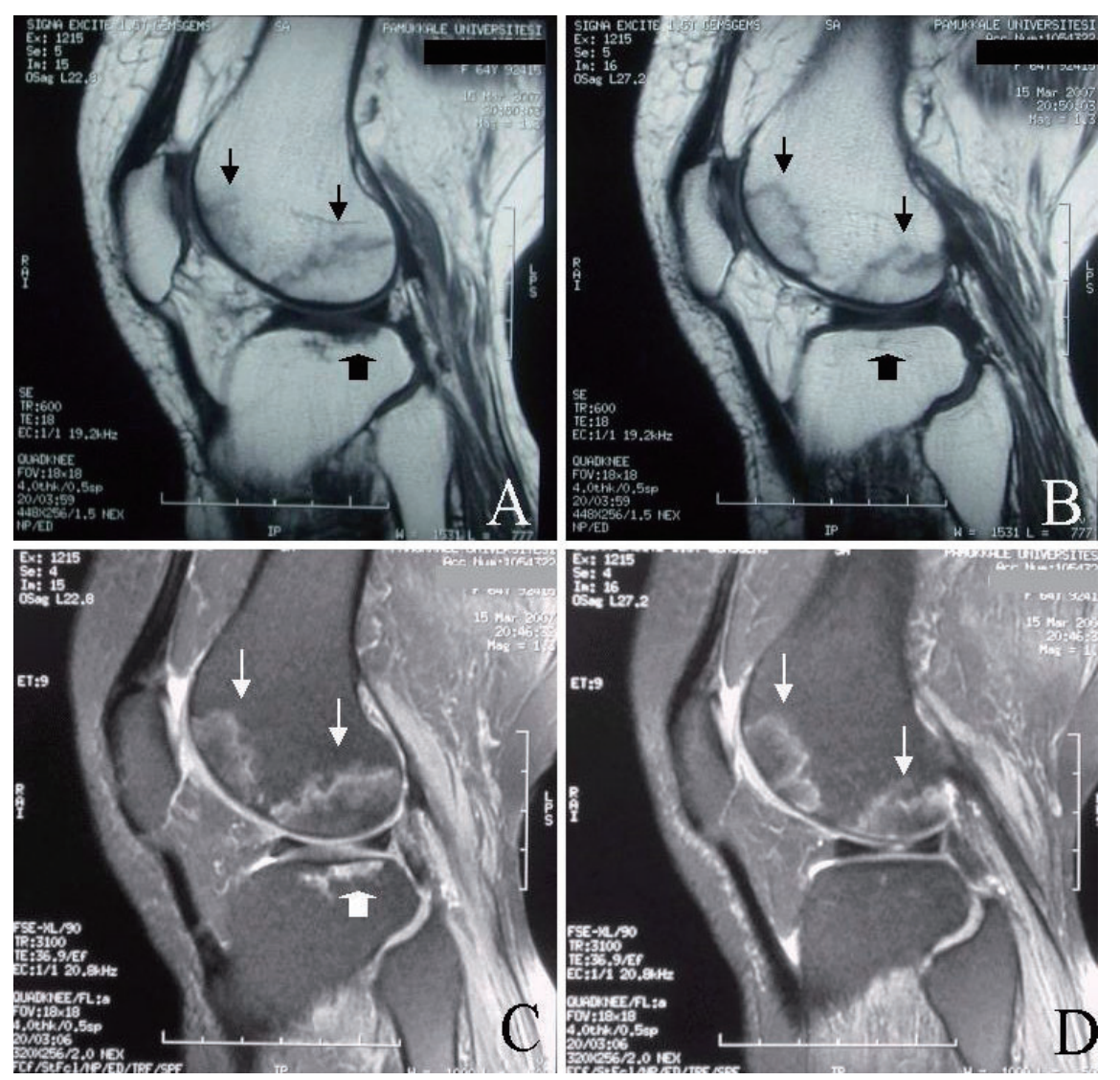

Figure 3. Sagittal T1-weighted (A-B) and T2-weighted (C-D) MR images of left knee show welldemarcated areas of osteonecrosis in lateral condyle (small arrows). MR images of left knee show area of subchondral bone marrow edema and necrosis in tibial plateau (large arrows).

steroids in very high short-term doses, in low long-term doses, or even by intra-articular injection (10-12). Incidence rates are highly variable ranging from $3 \%$ to over $25 \%$. There is no clear dose-response relationship between steroid therapy and the risk of AVN. It appears that risk increase depends on both the steroid dose and the duration of treatment, but it is difficult to predict in which patients osteonecrosis will develop $(1,11,13)$.

Some investigators have found that the daily dose of steroid may be the only risk factor. The risk of AVN, increases by $4 \%$ to $6 \%$ for every $10 \mathrm{mg} /$ day increase in oral prednisone treatment during the first 6 months of therapy. There is evidence that the duration of steroid use is an important factor in the subsequent development of AVN. Bolus doses of steroid usually pose little risk of AVN (14).

The cumulative dose of steroid is important with respect to the risk of developing osteonecrosis. Morris et al demonstrated that reducing the cumulative steroid dose in renal transplant patients over two years decreased the risk of developing AVN (15). In another article, cumulative steroid dose is indicated to be the most important risk factor for osteonecrosis. In the study, it was stated that the risk of osteonecrosis was $0.6 \%$ for patients receiving less than $3 \mathrm{~g}$ and $13 \%$ for patients receiving more than $3 \mathrm{~g}(8)$. In the present patient, total intake of steroid dose was over $3 \mathrm{~g}$, which had been a severe risk factor for AVN development.
The interval between steroid administration and the onset of symptoms is frequently within 6 or 8 months after starting the steroid therapy. It rarely occurs in less than 6 months and may take more than 3 years (10). Nagasawa et al described AVN due to steroid use in the first three months of the treatment as 'very early' (5). In the present case, symptoms started 15 days after the initiation of steroid therapy, and the patient was diagnosed with AVN at the 45th day of treatment. As it was described in the literature, this diagnosis was made at a very early stage.

Direct radiography is not diagnostic for osteonecrosis until a relatively late stage of disease. At present, MR imaging is the diagnostic study of choice with respect to both sensitivity and specificity because it can define early radiographically negative AVN in asymptomatic individuals $(8,16)$. In our case, AVN which could not be diagnosed by plain radiography is diagnosed accurately by MR imaging.

The most common site of steroid-induced AVN is the femoral head. The distal femur and proximal tibia, humeral head, talus and capitellum may also be involved (8). Concurrent hip and knee involvement in steroid-induced AVN was described only in 13 patients with severe acute respiratory syndrome, non-Hodgkin lymphoma, acute lymphoblastic leukemia, and ulcerative colitis (6-9).

Early diagnosis of osteonecrosis, especially in younger patients, allows conservative interventions including joint 
rest, nonsteroidal anti-inflammatory drugs, range of motion exercises, strengthening of the muscle surrounding the affected bone, limitation of weight-bearing across the affected joint(s), and mobility training (17). Conservative management may prevent collapse of subchondral bone, avert progression of disease, and delay any surgical procedures until absolutely necessary (1). Progression of the necrosis may lead to subchondral bone collapse, joint incongruity, subsequent joint destruction, and the need for total knee or hip replacement (18).

\section{Conclusion}

AVN is a devastating untoward effect of a ubiquitous medication that often leads to functional impairment and severe disability. In the literature, steroid-induced AVN is reported to be rare in ITP patients. In addition, concomitance of both hip and knee AVN is rare and also very early diagnosed AVN is exceptional. This case report is the first to define concurrent AVN of hip-and-knee at a very early stage due to steroid use in a patient with ITP.

Physicians should suspect this condition in patients with a history of steroid use who complain of severe joint pain. A prompt diagnosis can be made with MRI, and appropriate management may ensue.

\section{References}

1. Braverman DL, Lachmann EA, Nagler W. Avascular necrosis of bilateral secondary to corticosteroid enemas. Arch Phys Med Rehabil 79: 449-451, 1998.

2. Chang CC, Greenspan A, Gershwin ME. Osteonecrosis: current perspectives on pathogenesis and treatment. Semin Arthritis Rheumatol 23: 47-69, 1993.

3. Usui $M$, Inoue $H$, Yukihiro $S$, Abe N. Femoral neck fracture following avascular necrosis of the femoral head. Acta Med Okayama 50 (2): 111-117, 1996.

4. Havel PE, Ebraheim NA, Jackson T. Steroid-induced bilateral avascular necrosis of the lateral femoral condyles. A case report. Clin Orthop Rel Res 243: 166-168, 1989.

5. Nagasawa K, Tada Y, Koarada S, et al. Very early development of steroid-associated osteonecrosis of femoral head in systemic lupus erythematosus: prospective study by MRI. Lupus 14: 385-390, 2005.

6. Hong N, Du XK. Avascular necrosis of bone in severe acute respiratory syndrome. Clin Radiol 59: 602-608, 2004.

7. Sawicka-Zukowska M, Kajdas L, Muszynska-Roslan K, Krawczuk-Rybak M, Sonta-Jakimczyk D, Szczepanski T. Avascular necrosis - An antineoplastic treatment related toxicity: The experiences of two institutions. Pediatr Hematol Oncol 23: 625-629, 2006.

8. Griffith JF, Antonio GE, Kumta SM, et al. Osteonecrosis of hip and knee in patients with severe acute respiratory syndrome treated with steroids. Radiology 235: 168-175, 2005.

9. Vidal Casariego A, de la Cuerda Compés C, Bretón Lesmes I, et al. Avascular necrosis of both hips and knees in a patient with ulcerative colitis treated for a long term with high-dose corticosteroids. Nutr Hosp 21 (1): 109-112, 2006 (Spanish).
10. Metselaar HJ, van Steenberge EJP, Bijnen AB, Jeekel JJ, van Linge B, Weimar W. Incidence of osteonecrosis after renal transplantation. Acta Orthop Scand 56: 413-415, 1985.

11. O'Brien TJ, Mack GR. Multifocal osteonecrosis after short-term high-dose corticosteroid therapy. Clin Orthop Rel Res 279: 176179, 1992.

12. Laroche M, Arlet J, Mazieres B. Osteonecrosis of the femoral and humeral heads after intraarticular corticosteroid injections. J Romatol 17: 549-551, 1990.

13. Zizic TM, Marcoux C, Hungerford DS, Densereau JV, Stevens MB. Corticosteroid therapy associated with ischemic necrosis of bone in systemic lupus erythematosus. Am J Med 79: 596-604, 1985.

14. Felson DT, Anderson JJ. Across-study evaluation of association between steroid dose and bolus steroids and avascular necrosis of bone. Lancet 1 (8538): 902-906, 1987.

15. Morris PJ, Chan L, French ME, Ting A. Low dose oral prednisolone in renal transplantation. Lancet 1: 525-527, 1982.

16. Stevens K, Tao C, Lee SU, et al. Subchondral fractures in osteonecrosis of the femoral head: comparison of radiography, CT, and MR imaging. AJR Am J Roentgenol 180: 363-368, 2003.

17. Motohashi M, Morii T, Koshino T. Clinical course of roentgenographic changes of osteonecrosis on the femoral condyle under conservative treatment. Clin Orthop 266: 156-161, 1991.

18. Seldes RM, Tan V, Duffy G, Rand JA, Lotke PA. Total knee arthroplasty for steroid-induced osteonecrosis. J Arthroplasty 14 (5): 533-537, 1999.

(C) 2008 The Japanese Society of Internal Medicine http://www.naika.or.jp/imindex.html 